\title{
Community Level Disadvantage and the Likelihood of First Ischemic Stroke
}

\author{
Bernadette Boden-Albala, ${ }^{1,2}$ Eric T. Roberts, ${ }^{1,3}$ Harmon Moats, ${ }^{4}$ Hiba Arif, ${ }^{4}$ \\ Ralph L. Sacco, ${ }^{5}$ and Myunghee C. Paik ${ }^{6}$ \\ ${ }^{1}$ Division of Social Epidemiology, Department of Health Evidence and Policy, Mount Sinai School of Medicine, \\ New York, NY 10029, USA \\ ${ }^{2}$ Department of Neurology, Mount Sinai School of Medicine, New York, NY 10029, USA \\ ${ }^{3}$ Department of Epidemiology, Mailman School of Public Health, Columbia University, New York, NY 10032, USA \\ ${ }^{4}$ Department of Neurology, College of Physicians and Surgeons, Columbia University, New York, NY 10032, USA \\ ${ }^{5}$ Departments of Neurology, Epidemiology, Human Genetics, Miller School of Medicine, University of Miami, FL 33136, USA \\ ${ }^{6}$ Department of Biostatistics, College of Physicians and Surgeons, Columbia University, New York, NY 10032, USA
}

Correspondence should be addressed to Bernadette Boden-Albala, bernadette.boden-albala@mountsinai.org

Received 13 April 2012; Revised 27 September 2012; Accepted 28 September 2012

Academic Editor: Demosthenes Panagiotakos

Copyright () 2012 Bernadette Boden-Albala et al. This is an open access article distributed under the Creative Commons Attribution License, which permits unrestricted use, distribution, and reproduction in any medium, provided the original work is properly cited.

\begin{abstract}
Background and Purpose. Residing in "disadvantaged" communities may increase morbidity and mortality independent of individual social resources and biological factors. This study evaluates the impact of population-level disadvantage on incident ischemic stroke likelihood in a multiethnic urban population. Methods. A population based case-control study was conducted in an ethnically diverse community of New York. First ischemic stroke cases and community controls were enrolled and a stroke risk assessment performed. Data regarding population level economic indicators for each census tract was assembled using geocoding. Census variables were also grouped together to define a broader measure of collective disadvantage. We evaluated the likelihood of stroke for population-level variables controlling for individual social (education, social isolation, and insurance) and vascular risk factors. Results. We age-, sex-, and race-ethnicity-matched 687 incident ischemic stroke cases to 1153 community controls. The mean age was 69 years: $60 \%$ women; $22 \%$ white, $28 \%$ black, and 50\% Hispanic. After adjustment, the index of community level disadvantage (OR 2.0, 95\% CI 1.7-2.1) was associated with increased stroke likelihood overall and among all three raceethnic groups. Conclusion. Social inequalities measured by census tract data including indices of community disadvantage confer a significant likelihood of ischemic stroke independent of conventional risk factors.
\end{abstract}

\section{Introduction}

Stroke continues to burden health systems in all countries. Much work has clarified key biological risk factors including hypertension, diabetes, and coronary artery disease as well as lifestyle factors including smoking, diet, and physical activity. Increasingly epidemiology has investigated measures of socioeconomic status (SES) including education, income, and occupation and indeed a number of papers have documented associations between SES and overall and diseasespecific morbidity and mortality, including stroke [1-13].
SES may be a major contributor to race/ethnic and other disparities. A recent paper suggested that only $50 \%$ of the black-white stroke disparity is explained by biologic risk factors and measures of individual SES [3].

Beyond individual measures of SES, area measures of SES may contribute to rates of disease $[4,10,11]$. However, little work has investigated the association between area measures of SES controlling for individual measures of SES. Area level SES may be an important contributor to stroke disparities including by race/ethnicity. In this analysis, we sought to investigate the association of population-level disadvantage 
with incidence of first ischemic stroke in a model, which adjusts for individual level social and biological risk factors.

\section{Methods}

The Northern Manhattan Stroke Study (NOMAS) is a prospective, population-based incidence and case-control study designed to document the incidence of stroke, identify novel stroke risk factors, and document stroke prognosis in a multiethnic urban community. Northern Manhattan is home to approximately 260000 people, of which $63 \%$ are Hispanics, 20\% blacks, and 15\% whites. Overall, the Northern Manhattan area has a large population of immigrants (70\%), a high fertility rate (91.2 per 1000 women), and a significant proportion of households living below poverty level (28.6\%) [14]. The institutional review boards of Columbia-Presbyterian Medical Center and other area hospitals approved the study and written informed consent was obtained at the time of the in-person visit.

2.1. Selection of Cases. Methods for case detection in NOMAS utilizing a large comprehensive community-based stroke surveillance system have been previously described $[15,16]$. Briefly, 687 incident cases were prospectively enrolled over a 4-year period based on the following criteria: (1) diagnosed as having a first cerebral infarction; (2) older than 39 years at onset of stroke; (3) a resident of Northern Manhattan in a household with a telephone. During prospective surveillance process, trained research assistant used chart abstraction and patient and physician interview to document first onset of incident ischemic stroke verifying any clinical history or exam suspicious of prior stroke. As part of the baseline enrollment, controls were also screened and examined for previous stroke history.

2.2. Selection of Controls. Methods of control recruitment and enrollment have also been described in previous publications [16]. Subjects were identified by random digit dialing utilizing dual frame sampling. Interviews were conducted by Audits and Surveys Inc., using trained bilingual interviewers. The telephone response rate was $91 \%$ (9\% refused to be screened), and $87 \%$ of those eligible indicated that they were willing to participate in the study. Control subjects were recruited from the telephone sample to have an in-person baseline assessment. The enrollment response rate was 75\%; therefore, the overall response rate was $68 \%$ (telephone response $\times$ enrollment response). Controls were randomly selected from cells matched to the accumulating case group by age, gender, and race-ethnicity and were recontacted by the NOMAS staff, and invited to participate in the study. Appointments were made for in-person evaluations at the hospital or home for those who could not come in-person (7\% were done at home). The overall response rate for selected and matched controls was 75\%. Our objective was to match 2 control subjects to each stroke case. An $n: m$ matching strategy ( $n$ cases and $m$ controls) was utilized so that every case was matched in a strata with a control. Approximately $80 \%$ of the cases were matched to two controls (1:2 matching), and 12\% were matched to one control
(1:1 matching). Those cases for which no individually matched control was available $(8 \%)$ were matched to other case-control strata with similar values of matching variables. Hence, $8 \%$ of the cases were in strata with another case and at least one control, resulting in 2:1 or 2:2 matching. Over $58 \%$ of the stroke cases were women: $50 \%$ self-identified as Hispanic, 28\% identified as black, 22\% white, and 68\% were over 65 years of age. Similarly $60 \%$ of controls were women; $50 \%$ self-identified as Hispanic, 28\% identified as black, $22 \%$ identified as white, and $69 \%$ were over 65 years of age.

2.3. Baseline Evaluation. Race and ethnicity were defined by self-identification based upon a series of interview questions modeled after the US census [17]. Race was mutually exclusive and defined by six categories: "white, Black, Indian (American), Eskimo, Asian or Pacific Islander, and other." Ethnicity was subdivided as Hispanic or non-Hispanic based on the answer to the question: "Are you of Spanish/Hispanic origin?" Race-ethnic groupings were mutually exclusive. All participants responding affirmatively to being of Spanish origin or identifying as Hispanic were classified as Hispanic. A variety of reliability and validity sub-studies were performed to assess the telephone interview and in-person data collection instruments [18].

Participants were classified as hypertensive (HTN) if the systolic blood pressure recording was $\geq 140 \mathrm{~mm} \mathrm{Hg}$, or the diastolic blood pressure recording was $\geq 90 \mathrm{~mm} \mathrm{Hg}$ based on the average of the two blood pressure measurements; or a patient's self-report of a history of hypertension or use of an antihypertensive agent. Diabetes mellitus (DM) was defined by a fasting glucose greater than $126 \mathrm{mg} / \mathrm{dL}(7.7 \mathrm{mmol} / \mathrm{L})$ or the patient's self-report of such a history, or medication. Physical inactivity (Inactivity) was categorized as reporting no participation in "usual" leisure physical activities (walking, jogging, etc.) versus the participation in leisure activities [19]. Cardiac disease (CAD) was defined as a history of a heart attack, angina, heart surgery, cardiac catheterization, or cardiac medications.

Individual level social resources included education as defined by years of education completed. Education was further dichotomized into those who had completed high school versus those who had not received a high school diploma. Health care access was defined by insurance type. Mutually exclusive insurance categories were defined as no insurance (NINS), private insurance (PVT), Medicare only (MCR), and Medicaid (MCD) \pm Medicare. Social support was defined by marital status, and social isolation. We defined social isolation as "knowing less than 3 people well enough to visit within their homes." We defined acculturation as residency in the community greater than 5 years.

2.4. Census Tract Data. Census tract data from the 2000 US Census Bureau was ascertained using the Census tract street locator [20]. This application allows users to look up specific street addresses within a specific ZIP code. Exact address locations were used to identify the particular census tract for all subjects. Information regarding populationlevel economic and social indicators was then downloaded from each census tract. The two population-level economic 
indicators were (1) median household income, and (2) per capita income.

In addition to census level economic indicators, we adapted a validated index of community disadvantage obtained from census variables to define broader community level social resources [21]. Community disadvantage was defined as the proportion of the population below poverty level $(0.93)+$ the proportion of adults on public assistance $(0.94)+$ the proportion of female head of household + single head of household $(0.93)+$ the proportion of unemployed $(0.86)+$ the proportion of children in population (0.94) [22]

2.5. Statistics. All statistical analysis was completed using the SAS software (SAS Institute, Carey, NC). Baseline categorical data was compared with the use of the chi-square test. Student's $t$-test was used to compare mean age between cases and controls. Univariate odds ratios (ORs) were calculated for all population level variables using conditional logistic regression. Multivariable analysis was performed on all variables demonstrating a level of significance $(P<0.05)$, adjusting for biological stroke risk factors (HTN, DM, CAD and inactivity). Finally, an overall multivariate analysis was performed, adjusting for conventional stroke risk factors and individual social conditions including acculturation, insurance, social isolation and requiring a home health aid. Models were further stratified by race-ethnicity.

\section{Results}

Analyses were conducted on 687 first ischemic stroke cases and 1153 controls enrolled in the Northern Manhattan Stroke Study. Among the cases the mean age was $69 \pm 12$ years; $60 \%$ were women and $22 \%$ whites, $28 \%$ black and $50 \%$ Hispanic. Table 1 illustrates the demographic and risk factor profile of both cases and controls. Cases were significantly more likely to be hypertensive, diabetic, physically inactive, and have cardiac disease. Further, stroke cases were significantly less likely to have completed high school, more likely to be socially isolated, require help at home, and have Medicaid insurance.

We identified 41 census tracts representing subjects from the five zip code areas of Northern Manhattan. Blacks and Hispanics live together in over 50\% (21/41) of census tract areas, and Hispanics and whites lived together in 12 census tract areas. In 2000, the overall median per capita income for the Northern Manhattan census tracts was \$13,601.2 (range $\$ 12,144$ to $\$ 15,640$ ). The median household income was $\$ 27,862.8$ (range $\$ 24,285$ to $\$ 31,348$ ). Overall, census tracts of stroke cases had lower mean per capita and mean household income levels than community-based controls. The overall mean Disadvantage Index for the study area was 1.70 , with stroke cases (Disadvantage Index $=1.8$ ) living in census tract areas with greater community disadvantage than controls (Disadvantage Index $=1.5$ ).

The relationship between population-level economic indicators and individual social resources was investigated. Although the direction of association between these measures was in the expected direction, no strong colinear associations were found.
Univariate conditional logistic analysis was used to obtain unadjusted OR's (95\% CI) for each of the census tract economic indicators (Table 2). Lower per capita income was significantly associated with increased stroke likelihood while median household income was not. Multivariable analysis was performed for per capita income, adjusting for conventional stroke risk factors and individual social conditions (Table 2). After adjustment, per capita income remained associated with decreased stroke events (OR 0.8, 95\% CI 0.6-1.0). Per capita income was inversely associated with stroke outcomes demonstrating a $20 \%$ reduction in incident stroke events for each $\$ 10,000$ measured at the community aggregate. When stratified by race-ethnicity the results were not statistically significance.

A final multivariate model incorporated vascular risk factors, individual level social resources and the community disadvantage index. Per capita income was not placed in the model because it was strongly correlated with the community disadvantage index. As demonstrated in Figure 1, community disadvantage conferred an additional significant likelihood of ischemic stroke (OR 2.0 (1.7-2.1)) after adjusting for individual biological risk factors including CAD, HTN, $\mathrm{DM}$, and inactivity as well as individual social determinants including social isolation, Medicaid insurance, less than completed high school education, and having a home health aid. The relationship persisted among all three race-ethnic groups in models stratified by race-ethnicity: whites (OR 2.2, 95\% CI 1.7-2.7), blacks (OR 1.7, 95\% CI 1.5-2.0), and Hispanics (OR 1.6, 95\% CI 1.4-1.7).

\section{Discussion}

This study supports the hypothesis that population level social indicators are related to increased likelihood of first ischemic stroke even after adjustment for both conventional risk factors and individual social conditions. Population measures of wealth and overall community disadvantage were both associated with an increased likelihood of stroke and the measure of community disadvantage was associated with an increased likelihood of stroke among all race/ethnic groups.

Other literature documents the inverse relationship between socioeconomic indicators and overall and diseasespecific mortality, including cardiovascular disease and stroke consistent with our results $[3-6,9,10,12,13,23,24]$. However, many of these studies have been criticized for only using aggregate data $[25,26]$ and our study utilized both individual and area measures of SES providing confidence that both measures of SES contribute independently to the likelihood of stroke.

Few studies link individual risk factors, social resources, and population level community factors. There is sparse data regarding the influence of population level economic factors on overall stroke mortality. A study from Canada reports an inverse relationship between income and stroke mortality, finding that each $\$ 10,000$ increase in median household income per census tract results in a 9\% decrease in 30-day mortality poststroke, and a $5 \%$ decrease in 1-year mortality [6]. These data suggest that despite relative equity 
TABLE 1: Demographics, risk factor prevalence, and distribution of social resources among ischemic stroke cases and age-, sex-, and race/ethnicity-matched controls: The Northern Manhattan Stroke Study.

\begin{tabular}{|c|c|c|c|}
\hline & Definitions & $\begin{array}{c}\text { Cases } \\
N=687 \\
N(\%)\end{array}$ & $\begin{array}{c}\text { Controls } \\
N=1153 \\
N(\%)\end{array}$ \\
\hline \multicolumn{4}{|l|}{ Sociodemographics } \\
\hline \multirow{2}{*}{ Age } & $\geq 65 \mathrm{yrs}$ & $467(68)$ & $796(69)$ \\
\hline & Mean age & $68.2 \pm 11$ yrs & $69.6 \pm 12$ yrs \\
\hline Gender & Women & $398(58)$ & $692(60)$ \\
\hline \multicolumn{4}{|l|}{ Race-ethnicity } \\
\hline & White & $151(22)$ & $254(22)$ \\
\hline & Black & $192(28)$ & $323(28)$ \\
\hline & Hispanic & $343(50)$ & $577(50)$ \\
\hline \multicolumn{4}{|l|}{ Vascular risk factors } \\
\hline Hypertension & $\mathrm{SBP} \geq 140, \mathrm{DBP} \geq 90$, or $\mathrm{HX}$ & $488(71)^{*}$ & $699(52)$ \\
\hline Diabetes & $\mathrm{FBS} \geq 126$ or $\mathrm{HX}$ & $206(30)^{*}$ & $184(16)$ \\
\hline Cardiac disease & HX MI, angina, cardiac meds, or surgery & $275(40)$ & $231(20)$ \\
\hline Physical Inactivity & No reported activity & $330(48)^{*}$ & $254(22)$ \\
\hline \multicolumn{4}{|l|}{ Social resources } \\
\hline Occupation & Unskilled & $304(44)$ & $461(40)$ \\
\hline \multirow[t]{3}{*}{ Education } & High School Graduate & $222(33)^{*}$ & $566(49)$ \\
\hline & Receiving home help & $139(21)^{*}$ & $128(11)$ \\
\hline & Insurance & & \\
\hline \multirow[t]{3}{*}{ Health Access } & Medicare & $443(66)$ & $796(69)$ \\
\hline & Medicaid & $298(44)^{*}$ & $339(29)$ \\
\hline & Private & $220(33)^{*}$ & $543(47)$ \\
\hline Acculturation & $>5$ years in community & $600(87)^{*}$ & $1096(95)$ \\
\hline \multirow{2}{*}{ Social Support } & Currently married & $224(33)$ & $343(30)$ \\
\hline & Social Isolation (less than 3 friends) & $172(25)^{*}$ & $186(16)$ \\
\hline
\end{tabular}

${ }^{*}$ Comparison between unmatched cases and controls statistically significant at $P<0.05$.

TABLe 2: Census tract indicators associated with likelihood of incident stroke: The Northern Manhattan Stroke Study.

\begin{tabular}{lcc}
\hline & \multicolumn{2}{c}{ Census indicators } \\
& Model for annual household income & Model for annual per capita income \\
& OR, 95\% CI & OR, 95\% CI \\
\hline Household income (per \$10,000) & $0.9,0.7-1.1$ & $0.8,0.6-0.9^{*}$ \\
Per capita income (per \$10,000) & NA & $2.1,1.6-2.9^{*}$ \\
Hypertension Hx & $2.1,1.5-2.8^{*}$ & $1.3,1.2-1.4^{*}$ \\
Diabetes Hx & $1.3,1.2-1.5^{*}$ & $1.7,1.3-2.2^{*}$ \\
Coronary disease Hx & $1.7,1.2-2.2^{*}$ & $2.1,1.7-2.7^{*}$ \\
Physical inactivity & $2.1,2.1-2.7^{*}$ & $1.1,1.0-1.3$ \\
Social isolation $(<3$ friends) & $1.2,1.0-1.3$ & $1.1,0.8-1.8$ \\
Medicaid insurance & $1.1,0.8-1.5$ & $1.3,1.2-1.5^{*}$ \\
Not completed high school & $1.3,1.3-1.5^{*}$ & $1.5,1.1-2.1^{*}$ \\
Home health aid & $1.5,1.1-2.1^{*}$ & $0.9,0.9-0.9^{*}$ \\
Years in community & $0.9,0.9-1.0^{*}$ & \\
\hline
\end{tabular}

* Indicates $P$ value $<0.05$.

${ }^{\dagger}$ Matched for age, gender and race-ethnicity. 


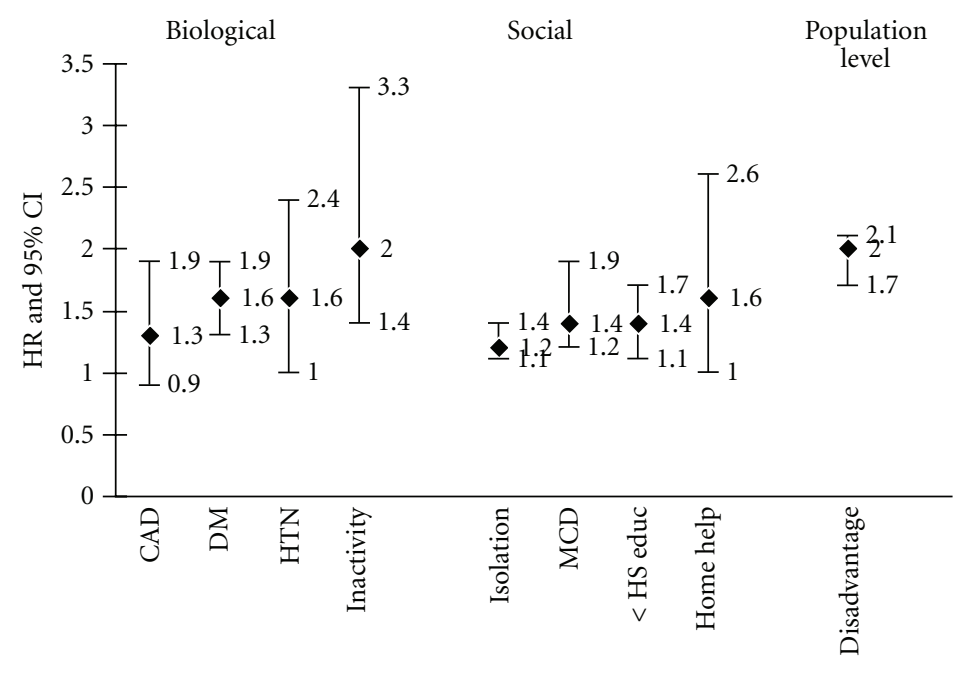

Matched for age, gender, and
race-ethnicity

Figure 1: Multivariable stroke model utilizing biological, social, and population level risk factors.

in health care access, disparities in mortality by income continued to exist. In our study, we report an inverse significant relationship between per capita income and likelihood of first ischemic stroke even after adjustment for traditional vascular risk factors and individual social resources including educational level, insurance status, and social support.

Socioeconomic status is reported to influence stroke risk and may be confounded by race-ethnicity. One study found that low SES was associated with increased stroke mortality $(P \leq 0.0001)$, and accounted for $14 \%$ to $46 \%$ of the excess black stroke risk $(P \leq 0.05)$ [27]. Another study focused on income inequality and overall mortality suggested that whites have a greater mortality risk associated with economic inequality compared to blacks [28]. One question is whether race-ethnicity may actually be a proxy for detrimental neighborhood characteristics. The Brain Attack Surveillance in Corpus Christi Project (BASIC) study found that raceethnicity was not associated with increase stroke score but that neighborhood characteristics were significantly associated with stroke-risk score in certain gender and age groups [29]. After stratification by race/ethnicity the association between per capita income and likelihood of stroke was not statistically significant; however, we observed strong associations between our community disadvantage index and the likelihood of stroke. While a lack of statistical power cannot be ruled out, there are problems with the measurement of income that may explain our results. Retirees are on a fixed income, and therefore the older age of stroke onset may attenuate the effect of per capita income among whites and perhaps blacks. Further, there are significant amounts of undocumented income in low-income neighborhoods [21].

We report that an index of community disadvantage was significantly related to likelihood of ischemic stroke. First ischemic stroke increased twofold among individuals living in communities that were more "disadvantaged" even after adjustment for a large number of conventional and individual social risk factors. Community disadvantage is thought to be associated with poorer health status through multiple psychosocial and physiological mechanisms. Sampson suggests that in the US the "ecological differentiation by social class, race, and health is a robust and increasingly occurring at multiple levels of geography whether it be neighborhoods, local communities, or states" [11, pS56]. Disparities in educational levels, employment training, health care access, and housing are likely to exist in highly disadvantaged communities. Further, individual behaviors may be influenced by the behaviors of those around them [30]. Indeed, it has been suggested that the health of older adults and of women that are at home with children may be particularly affected if they live in communities with unsupportive social and service environments [30].

Although we found no association between depressive symptoms and ischemic stroke, individuals within these disadvantaged communities may experience high levels of stress and fear, which may be associated with increased levels of blood pressure, glutocorticoids, and other physiological responses to stress $[11,31,32]$. Poorer neighborhoods may be more likely to have features which promote unhealthy lifestyles, including a higher density of alcohol outlets and limited high-priced healthy foods $[33,34]$.

Further, the built environment and the role of physical aspects of neighborhoods may be characterized by physical disorder and deterioration as well as a high rate of social disorder and unrest due to a lack of capital infusion [35]. Rundle and colleagues report an association between urban form including bus and subway stop density, population density, and intersection density with body size in New York City including Northern Manhattan [36]. Indeed, studies indicate that attractive, aesthetically pleasing settings are more conducive to physical activity than areas that 
are unattractive and possibly dangerous [37]. This effect, though indirect, may be very strong: in one survey, $31 \%$ of low-income persons are afraid to walk or jog in their neighborhoods, compared to $15 \%$ of higher income persons [37]. Finally, communities which lack social cohesion, and social support, and where high levels of unemployment, and illiteracy exist are associated with deviant behavior and vandalism [38, 39].

Our data provides strong evidence to support the idea that the health of the individual within the community is influenced as much by community disadvantage as by the health and social status of the individual. While the predominant thinking about health is that individual knowledge, attitudes, perceptions and skill sets determine our health behaviors, the possibility that the conditions under which we live are equally responsible for increasing our risk of stroke disease, suggest that strategies for prevention may need to focus on community level issues.

If indeed as these results demonstrate, community level indicators are equally as important as individual level variables in increasing the likelihood of stroke incidence, how then do we think about intervention strategies? First, the results of this case-control analyses need to be replicated in prospective studies, and components of community level variables which can be manipulated need to be identified. Our measures of SES are generic reflections of social resources and do not point to specific policy interventions. Future work needs to elucidate the mechanisms by which SES contributes to morbidity and mortality. Finally, structural interventions might integrate risk reduction information and strategies into existing community services for "one-stop shopping" where, for example, unemployment filing and hypertension screening are combined.

Limitations do exist in examining the impact of census level data on individual stroke risk. Despite census tract differences in per capita income, and disadvantage, the Northern Manhattan community may be characterized as relatively homogenous especially with regard to economic indicators; these fall within a very narrow range. The nature of the population and relatively confined geographical location probably attenuate the true effect of social inequalities. Further, per capita income may be an inaccurate measure of economic solvency especially in a retired community. Unreported income, shifts in saving accounts, and transfer of property are more likely to occur in an elderly population. Better measures of wealth may have been able to capture other economic resources. Census tract data may also not be reflective of "true communities" - they are arbitrary barriers that were set up by record keepers. Despite this potential limitation, the distribution of race-ethnicity by census tracts does suggest that these "geographical boundaries" reflect smaller homogeneous enclaves. Other studies also concede that census tracts are valid measures in capturing neighborhoods [40]. Census tract boundaries may also change from census to census.

The inclusion criteria of having a telephone and or being able to be reached by phone through random digit dialing may well have excluded those most disenfranchised members of the underlying community. However, this exclusion pertains to both case and control enrollment and is unlikely to alter the results. Finally, NOMAS was powered on its initial aims which included the detection of differences in individual risk factors. We acknowledge that this analysis may well be underpowered.

\section{Conclusion}

Social inequalities in our society continue to exist, and their influence on mortality and morbidity continues to grow. In these analyses, social inequalities measured by census tract per capita income as well as by an index of community disadvantage confer an independent likelihood of ischemic stroke adjusting for conventional risk factors. These data suggest that we will not be able to measurably reduce stroke risk without emphasis on social and economic issues. Public health policy needs to focus its attention and resources on addressing issues of community level inequality.

\section{Acknowledgment}

This work was supported by a Grant from the National Institute of Neurological Disorders and Stroke (NINDS 29993).

\section{References}

[1] M. Casper, S. Wing, and D. Strogatz, "Variation in the magnitude of black-white differences in stroke mortality by community occupational structure," Journal of Epidemiology and Community Health, vol. 45, no. 4, pp. 302-306, 1991.

[2] J. J. Feldman, D. M. Makuc, J. C. Kleinman, and J. CornoniHuntley, "National trends in educational differentials in mortality," American Journal of Epidemiology, vol. 129, no. 5, pp. 919-933, 1989.

[3] G. Howard, M. Cushman, B. M. Kissela et al., "Traditional risk factors as the underlying cause of racial disparities in stroke: lessons from the half-full (empty?) glass," Stroke, vol. 42, no. 12, pp. 3369-3375, 2011.

[4] D. Jakovljević, C. Sarti, J. Sivenius et al., "Socioeconomic status and ischemic stroke: the FINMONICA stroke register," Stroke, vol. 32, no. 7, pp. 1492-1498, 2001.

[5] G. A. Kaplan and J. E. Keil, "Socioeconomic factors and cardiovascular disease: a review of the literature," Circulation, vol. 88, no. 4, pp. 1973-1998, 1993.

[6] M. K. Kapral, H. Wang, M. Mamdani, and J. V. Tu, "Effect of socioeconomic status on treatment and mortality after stroke," Stroke, vol. 33, no. 1, pp. 268-273, 2002.

[7] P. Kotler and D. L. Wingard, "The effect of occupational, marital and parental roles on mortality: the Alameda County study," American Journal of Public Health, vol. 79, no. 5, pp. 607-612, 1989.

[8] G. Pappas, S. Queen, W. Hadden, and G. Fisher, "The increasing disparity in mortality between socioeconomic groups in the United States, 1960 and 1986," New England Journal of Medicine, vol. 329, no. 2, pp. 103-109, 1993.

[9] F. R. Artalejo, P. Guallar-Castillón, J. L. Gutiérrez-Fisac, J. R. Banegas, and J. Del Rey Calero, "Socioeconomic level, sedentary lifestyle, and wine consumption as possible explanations for geographic distribution of cerebrovascular disease mortality in Spain," Stroke, vol. 28, no. 5, pp. 922-928, 1997. 
[10] C. E. Ross and J. Mirowsky, "Neighborhood disadvantage, disorder, and health," Journal of Health and Social Behavior, vol. 42 , no. 3, pp. 258-276, 2001.

[11] R. J. Sampson, "The neighborhood context of well-being," Perspectives in Biology and Medicine, vol. 46, supplement 3, pp. S53-S64, 2003.

[12] P. D. Sorlie, E. Backlund, and J. B. Keller, "US mortality by economic, demographic, and social characteristics: the National Longitudinal Mortality Study," American Journal of Public Health, vol. 85, no. 7, pp. 949-956, 1995.

[13] C. T. M. Van Rossum, H. Van De Mheen, M. M. B. Breteler, D. E. Grobbee, and J. P. Mackenbach, "Socioeconomic differences in stroke among Dutch elderly women: the Rotterdam Study," Stroke, vol. 30, no. 2, pp. 357-362, 1999.

[14] "United Hospital Fund," New York City Community Health Atlas, 2002, https://www.census.gov/census2000/states/ny .htm.

[15] R. L. Sacco, B. Boden-Albala, R. Gan et al., "Stroke incidence among white, black, and Hispanic residents of an urban community: the Northern Manhattan Stroke Study," American Journal of Epidemiology, vol. 147, no. 3, pp. 259-268, 1998.

[16] R. L. Sacco, M. Elkind, B. Boden-Albala et al., "The protective effect of moderate alcohol consumption on ischemic stroke," Journal of the American Medical Association, vol. 281, no. 1, pp. 53-60, 1999.

[17] Federal Registrar, Race and Ethnic Standards For Federal Statistics and Administrative Reporting, vol. 15, Washington, DC, USA, 1978.

[18] D. E. Kargman, R. L. Sacco, B. Boden-Albala, M. C. Paik, W. A. Hauser, and S. Shea, "Validity of telephone interview data for vascular disease risk factors in a racially mixed urban community: the northern Manhattan stroke study," Neuroepidemiology, vol. 18, no. 4, pp. 174-184, 1999.

[19] R. L. Sacco, R. Gan, B. Boden-Albala et al., "Leisure-time physical activity and ischemic stroke risk: the Northern Manhattan Stroke Study," Stroke, vol. 29, no. 2, pp. 380-387, 1998.

[20] "United States Census Bureau," The American Factfinder page, 2009, http://factfinder.census.gov/.

[21] D. C. Ompad, V. Nandi, M. Cerdá, N. Crawford, S. Galea, and D. Vlahov, "Beyond income: material resources among drug users in economically-disadvantaged New York City neighborhoods," Drug and Alcohol Dependence, vol. 120, no. 1-3, pp. 127-134, 2012.

[22] R. J. Sampson, S. W. Raudenbush, and F. Earls, "Neighborhoods and violent crime: a multilevel study of collective efficacy," Science, vol. 277, no. 5328, pp. 918-924, 1997.

[23] M. L. Casper, E. B. Barnett, D. L. Armstrong, W. H. Giles, and C. J. Blanton, "Social class and race disparities in premature stroke mortality among men in North Carolina," Annals of Epidemiology, vol. 7, no. 2, pp. 146-153, 1997.

[24] B. Modan and D. K. Wagener, "Some epidemiological aspects of stroke: mortality/morbidity trends, age, sex, race, socioeconomic status," Stroke, vol. 23, no. 9, pp. 1230-1236, 1992.

[25] K. Fiscella and P. Franks, "Poverty or income inequality as predictor of mortality: longitudinal cohort study," British Medical Journal, vol. 314, no. 7096, pp. 1724-1727, 1997.

[26] H. Gravelle, "How much of the relation between population mortality and unequal distribution of income is a statistical artefact?" British Medical Journal, vol. 316, no. 7128, pp. 382385, 1998.

[27] G. Howard, G. B. Russell, R. Anderson et al., "Role of social class in excess black stroke mortality," Stroke, vol. 26, no. 10, pp. 1759-1763, 1995.
[28] K. Lochner, E. Pamuk, D. Makuc, B. P. Kennedy, and I. Kawachi, "State-level income inequality and individual mortality risk: a prospective, multilevel study," American Journal of Public Health, vol. 91, no. 3, pp. 385-391, 2001.

[29] L. D. Lisabeth, A. V. Diez Roux, J. D. Escobar, M. A. Smith, and L. B. Morgenstern, "Neighborhood environment and risk of ischemic stroke: the Brain Attack Surveillance in Corpus Christi (BASIC) Project," American Journal of Epidemiology, vol. 165, no. 3, pp. 279-287, 2007.

[30] M. M. Weden, R. M. Carpiano, and S. A. Robert, "Subjective and objective neighborhood characteristics and adult health," Social Science and Medicine, vol. 66, no. 6, pp. 1256-1270, 2008.

[31] M. L. Ganz, "The relationship between external threats and smoking in Central Harlem," American Journal of Public Health, vol. 90, no. 3, pp. 367-371, 2000.

[32] M. J. Paschall and M. L. Hubbard, "Effects of neighborhood and family stressors on African American male adolescents' self-worth and propensity for violent behavior," Journal of Consulting and Clinical Psychology, vol. 66, no. 5, pp. 825-831, 1998.

[33] T. A. Laveist and J. M. Wallace, "Health risk and inequitable distribution of liquor stores in African American neighborhood," Social Science and Medicine, vol. 51, no. 4, pp. 613-617, 2000.

[34] S. Macintyre, S. Maciver, and A. Sooman, "Area, class and health: should we be focusing on places or people?" Journal of Social Policy, vol. 22, no. 2, pp. 213-234, 1993.

[35] M. Shaw, D. Gordon, D. Dorling, R. Mitchell, and G. Davey Smith, "Increasing mortality differentials by residential area level of poverty: Britain 1981-1997," Social Science and Medicine, vol. 51, no. 1, pp. 151-153, 2000.

[36] A. Rundle, A. V. Diez Roux, L. M. Freeman, D. Miller, K. M. Neckerman, and C. C. Weiss, "The urban built environment and obesity in New York City: a multilevel analysis," American Journal of Health Promotion, vol. 21, supplement 4, pp. 326334, 2007.

[37] P. Yankelovich, "Survey of barriers to physical activity and healthy eating habits," in Proceedings of the American Dietetic Association Conference, Chicago, Ill, USA, 1995.

[38] L. F. Berkman and L. Breslow, Health and Ways of Living: The Alameda County Study, Oxford University Press, New York, NY, USA, 1983.

[39] D. Kim, A. V. Diez Roux, C. I. Kiefe, I. Kawachi, and K. Liu, "Do neighborhood socioeconomic deprivation and low social cohesion predict coronary calcification? the CARDIA study," American Journal of Epidemiology, vol. 172, no. 3, pp. 288-298, 2010.

[40] E. T. Murray, A. V. Diez Roux, M. Carnethon, P. L. Lutsey, H. $\mathrm{Ni}$, and E. S. O'Meara, “Trajectories of neighborhood poverty and associations with subclinical atherosclerosis and associated risk factors," American Journal of Epidemiology, vol. 171, no. 10, pp. 1099-1108, 2010. 


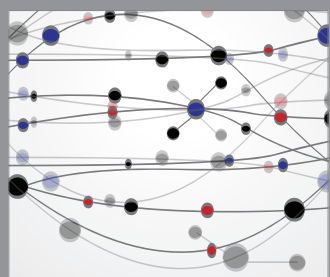

The Scientific World Journal
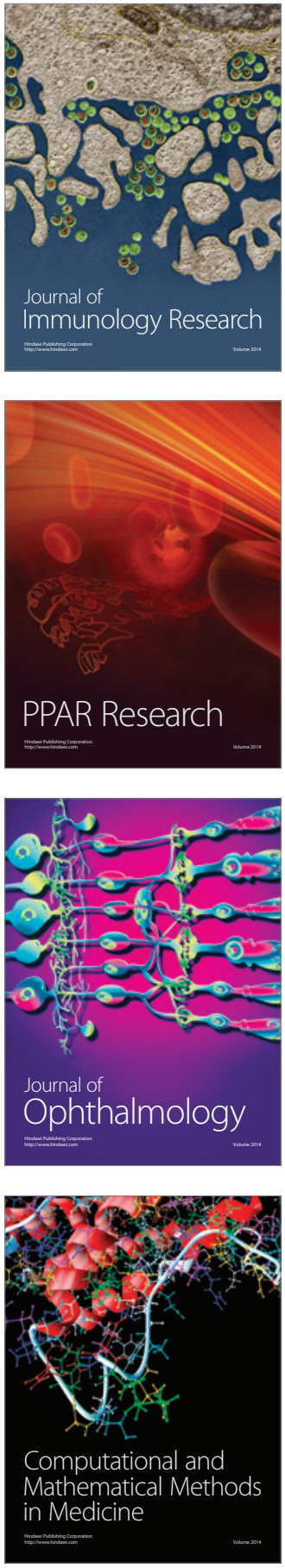

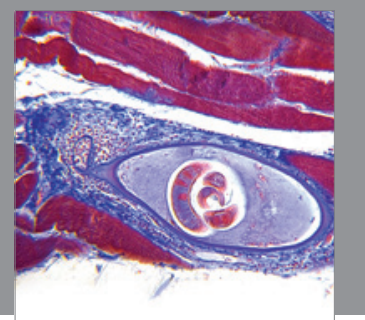

Gastroenterology

Research and Practice
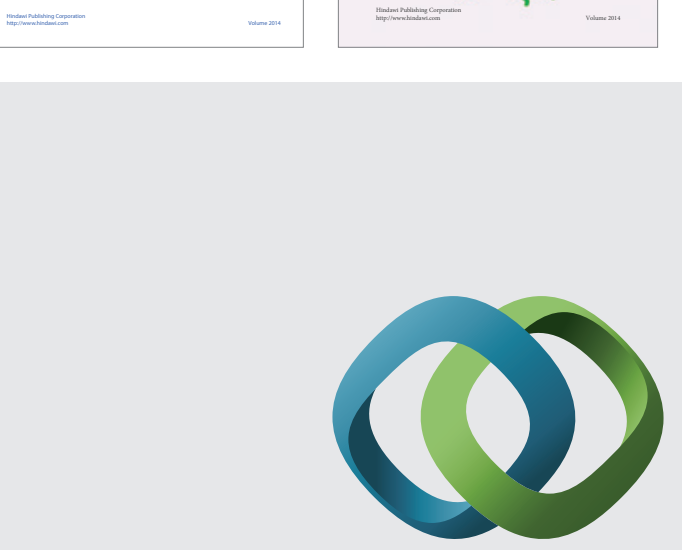

\section{Hindawi}

Submit your manuscripts at

http://www.hindawi.com
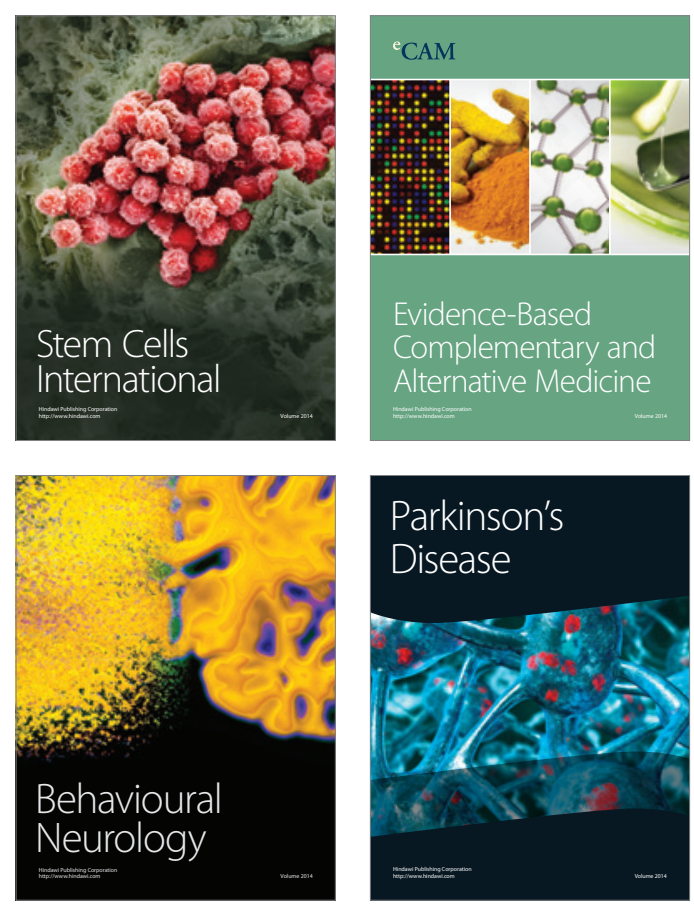

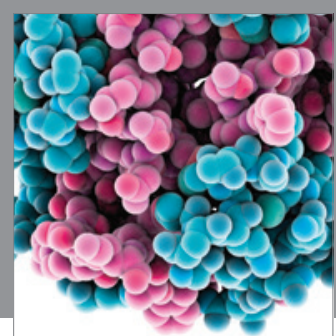

Journal of
Diabetes Research

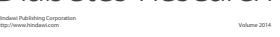

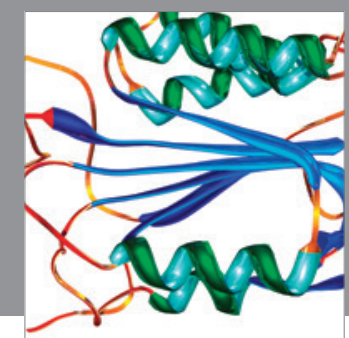

Disease Markers
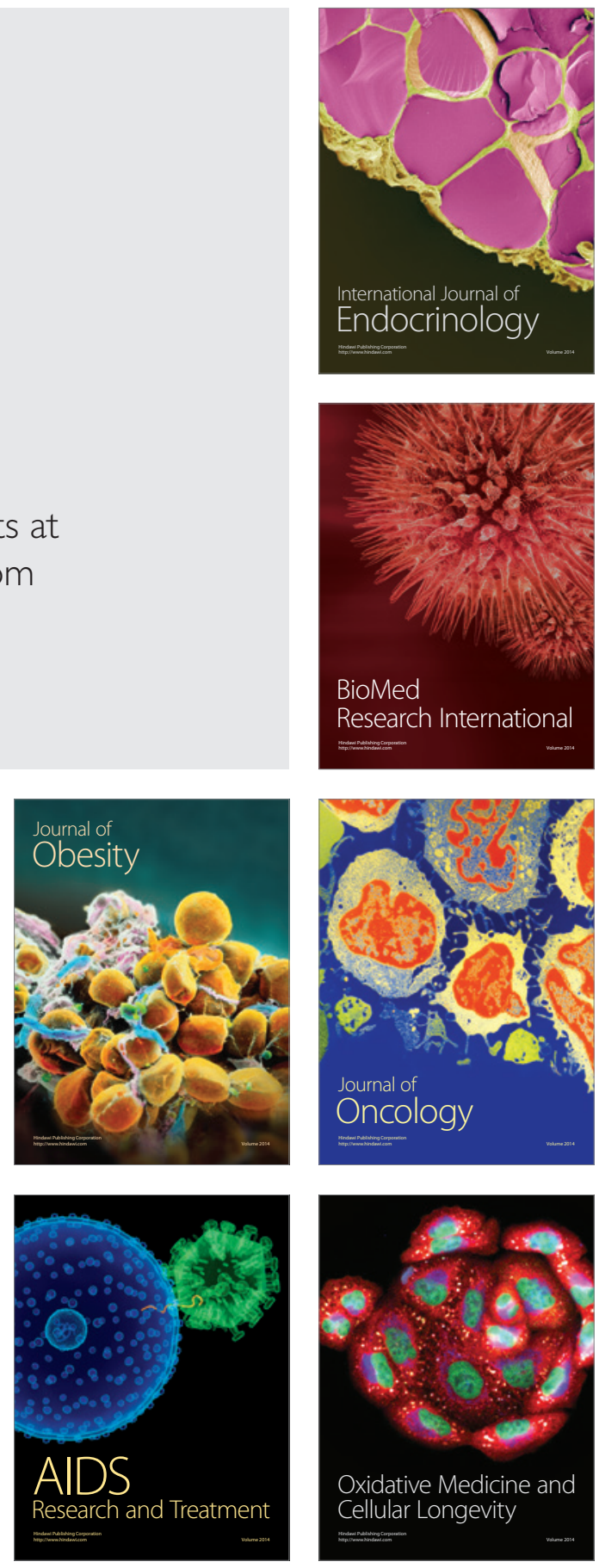